\title{
Electric-magnetic asymmetry of the dimension-2 condensate and the phases of Yang-Mills theory
}

\author{
M. N. Chernodub* \\ Laboratoire de Mathematiques et Physique Theorique CNRS UMR 6083, Fédération Denis \\ Poisson, Université de Tours, Parc de Grandmont, F37200, Tours, France \\ Institute of Theoretical and Experimental Physics ITEP, 117259 Moscow, Russia \\ E-mail: Maxim. Chernodubelmpt.univ-tours.fr
}

\section{E.-M. Ilgenfritz}

Institut für Physik, Humboldt-Universität zu Berlin, Newton-Str. 15, D-12489 Berlin, Germany Institut für Physik, Karl-Franzens-Universität Graz, Universitätsplatz 5, A-8010 Graz, Austria E-mail: ilgenfri@physik.hu-berlin.de

\begin{abstract}
We study the finite-temperature behavior of the $A^{2}$ condensate in the Landau gauge of $S U(2)$ Yang-Mills theory on the lattice in a wide range of temperatures. The asymmetry between the electric (temporal) and magnetic (spatial) components of this unconventional dimension- 2 condensate is a convenient, ultraviolet-finite quantity which possesses, as we demonstrate, unexpected properties. The low-temperature behavior of the condensate asymmetry suggests that the mass of the lowest thermal excitation in the condensate is unexpectedly low, about $200 \mathrm{MeV}$, which is much smaller than the glueball mass. The asymmetry is peaked at the phase transition, being a monotonically decreasing function in the deconfinement phase. A symmetric point is reached in the deconfinement phase at a temperature approximately equal twice the critical temperature. This behavior of the electric-magnetic asymmetry of the condensate separates the phase diagram of Yang-Mills theory into three regions. We suggest that these regions are associated with the condensed, liquid and gaseous states of the confining gluonic objects, the Abelian monopoles.
\end{abstract}

8th Conference Quark Confinement and the Hadron Spectrum

September 1-6 2008

Mainz, Germany

\footnotetext{
*Speaker.

†Partly supported by STINT Institutional Grant No. IG2004-2 025.

†E.-M. I. was supported by DFG through the Forschergruppe FOR 465 (Mu 932/2). He is grateful to the KarlFranzens-Universität Graz for the guest position he presently holds.
} 
Here we report results of our studies [1] of an unconventional dimension-2 condensate in Yang-Mills theory. An example of such a condensate is the nonperturbative part of the expectation value of the operator $A^{2}(x) \equiv A_{\mu}^{a}(x) A_{\mu}^{a}(x)$ in the (minimal) Landau gauge, defined by minimization of the $A^{2}$-operator with respect to $S U(2)$ gauge transformations. To our knowledge, the $A^{2}$ operator was first mentioned in Ref. [2]. Dimension-2 condensates - such as the $A^{2}$ condensate - were suggested to play a distinguished rôle in QCD phenomenology [3].

Thermal properties of the $A^{2}$ condensate may be revealed with the help of the electric-magnetic asymmetry of the condensate, $\Delta_{A^{2}}(T)=\left\langle g^{2} A_{4}^{2}\right\rangle-\left\langle g^{2} A_{i}^{2}\right\rangle / 3$, which is given by the difference in the thermal fluctuations of the electric (temporal) gluons, $A_{4}$, and magnetic (spatial) gluons, $A_{i}$.

The electric-magnetic asymmetry of the $A^{2}$-condensate in $S U(2)$ lattice gauge theory is shown in Figure 1. The results have very good scaling properties towards the continuum limit.
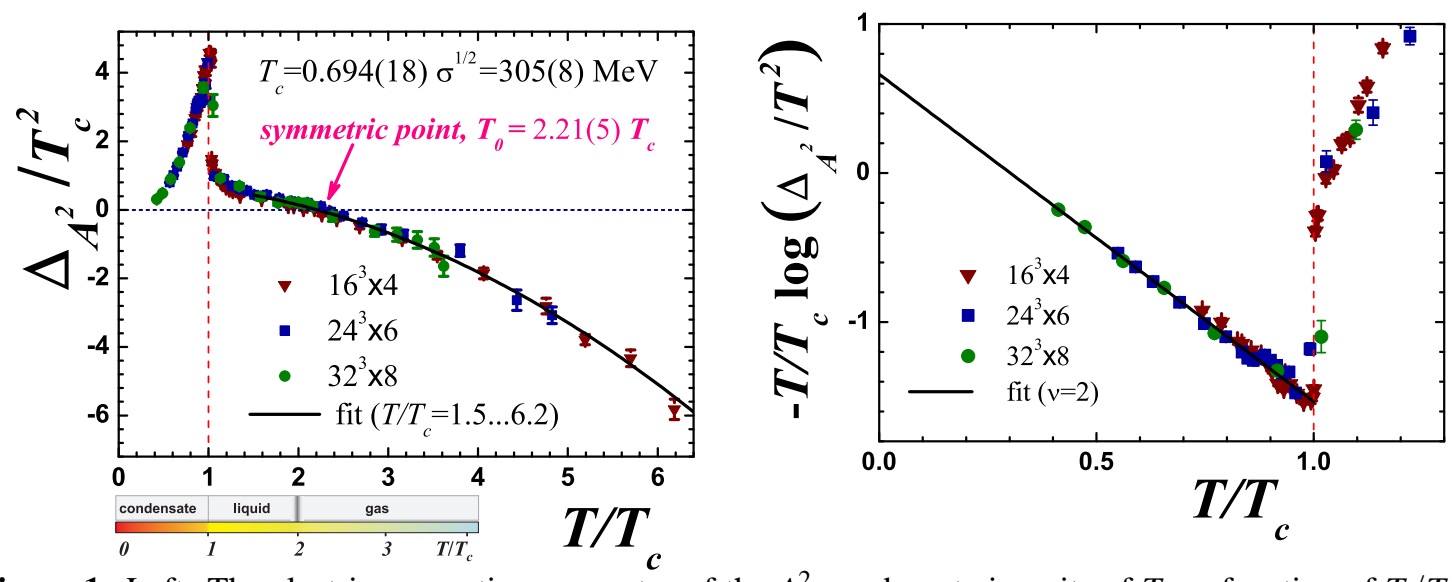

Figure 1: Left: The electric-magnetic asymmetry of the $A^{2}$-condensate in units of $T_{c}$ as function of $T / T_{c}$. The high-temperature fit $\Delta_{A^{2}}(T) \sim T^{2}$ is shown by the solid line. The relevant monopole phases are indicated explicitly [4] below, the symmetric point, $\Delta_{A^{2}}=0$, is shown by an arrow. Right: The best fit at low-temperature is $\Delta_{A^{2}}(T) \sim T^{2} \exp \left\{-m_{A^{2}} / T\right\}$ with an unexpectedly small value of $m_{A^{2}}=201(8) \mathrm{MeV}$.

Apart from the unexpected behavior of the asymmetry as a function of temperature (only the high- $T$ limit is understood [1] theoretically) there are two puzzles seen in Figure 1:

(i) The low- $T$ limit is controlled by the $A^{2}$-mass gap, $m_{A^{2}} \sim 200 \mathrm{MeV}$, which is much smaller than the lowest glueball mass, $m_{O^{++}} \sim 1.5 \mathrm{GeV}$. The $A^{2}$-mass gap is of the same order as $T_{c} \sim 300 \mathrm{MeV}$ indicating that the mass gap of the $A^{2}$-condensate may be relevant to the deconfinement transition. (ii) The asymmetry $\Delta_{A^{2}}(T)$ distinguishes three regions in the phase diagram: the confinement $\left(0<T<T_{c}\right)$, the strongly coupled deconfinement $\left(T_{c}<T \lesssim 2.2 T_{c}\right)$, and the high temperature region $\left(T \gtrsim 2.2 T_{c}\right.$ ). It is amusing to see these regions coinciding with the condensed, liquid and gaseous phases of the (topological) monopole component of the gluon plasma as suggested in Ref. [4].

\section{References}

[1] M. N. Chernodub and E.-M. Ilgenfritz, Phys.Rev. D78 (2008) 034036 [arXiv:0805.3714].

[2] R. Fukuda, Prog.Theor.Phys. 67 (1982) 648.

[3] K. G. Chetyrkin, S. Narison, and V. I. Zakharov, Nucl. Phys. B 550 (1999) 353 [hep-ph/9811275].

[4] M. N. Chernodub and V. I. Zakharov, Phys. Rev. Lett. 98 (2007) 082002 [arXiv:hep-ph/0611228]. 